\title{
High velocity impact of carbon composite plates: perforation simulation
}

\author{
E. Jacquet ${ }^{1}$, A. Rouquand ${ }^{1} \&$ O. Allix ${ }^{2}$ \\ ${ }^{1} D G A /$ Centre d'Etudes de Gramat, France \\ ${ }^{2}$ Laboratoire de Mécanique et Technologie, \\ ENS de Cachan/CNRS/Université Paris, France
}

\begin{abstract}
Aircraft structures are large and complex, generally constructed from thin sheet metal or composite materials. As a result, analysis and prediction of the behaviour of such structures when subjected to high-energy impacts is very complex. Computational design analysis techniques have the potential to provide a deeper understanding of the problem and hence enable the design of safer and more efficient airframes. With this end in mind, it is important to develop and identify good modelling techniques to ensure accurate representation of real life situations.

The mechanism of damage initiation and growth in layered composites subjected to high-velocity impacts is simulating using a damage mesomodel approach. The implementation of the model into the commercial finite element code ABAQUS via use-defined FORTRAN subroutines is described. The implemented model involves damage in both the mesoscale layer and an interface between the layers. In this paper, the first experimental and numerical results of high-velocity impacts of composite plates are described and compared, the advantages and disadvantages of numerical methods used are discussed and future developments are announced.
\end{abstract}

Keywords: ballistic impact, composites, models.

\section{Introduction}

Composite material has various advantages (high specific elasticity and strength, lightness in weight etc.), so it has been considered as the most prospective structural material in the aeronautic field for a long time. The topic of impact 
damage in laminated composite materials has been studied mostly for the lowvelocity impact caused by dropped tools or runway debris. Other studies deal with ballistic impacts caused by missiles or shell fragments with a velocity of less than $1000 \mathrm{~m} / \mathrm{s}$. This paper focuses on the ballistic impacts, ranging from $1000 \mathrm{~m} / \mathrm{s}$ to $3500 \mathrm{~m} / \mathrm{s}$, of thin carbon/epoxy laminated composite plates. Recent progress in materials modelling and numerical simulation of the impact response of fibre-reinforced composite plates are described. This paper makes use of continuum damage mechanics as developed by Ladevèze [1], as a framework within which in-ply and delamination failure may be modelled. The mesomodel of Ladevèze was developed originally for quasi-static loads, but it can be used because of the assumption that the damage mechanisms of laminated composites in dynamic are the same in static.

An important requirement for operational utilization is to develop numerical methods for the simulation of ballistic impacts on composites plates, thus the composite materials models need to be implemented and validated in finite element codes. Emphasis is placed in this paper on the implementation of the ply damage model and delamination models into the commercial explicit code Abaqus/Explicit and on the numerical difficulties of penetration phenomena.

\section{Damage mechanics and mesomodelling of laminates}

When dealing with composites, the key issue is the scale on which the model is constructed. This is also the scale on which the calculations have to be performed. On the one hand, the use of the microscale, besides numerous other difficulties, would raise the computing costs beyond reasonable limits. On the other hand, the use of macroscale would not enable a proper representation of the basic features of the laminate and of its deterioration mechanism. Moreover, for severe dynamic loading, the concept of homogenized material is meaningless. Therefore, it is necessary to define a scale on which the material can be described properly without going into excessive detail. A pragmatic approach consists of determining a characteristic length of the main damage mechanisms. For laminated composites, between the macroscale of the structure and the microscale of the single fibre, there is an intermediate modelling scale called the mesoscale. This scale is associated with the thickness of the layer and the thicknesses of the different interlaminar interfaces. On this scale, the main

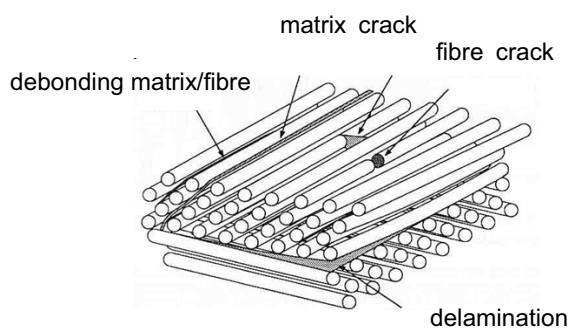

Figure 1: Main damage mechanisms. 
damage mechanisms (delamination, matrix microcracking, fibre/matrix debonding and matrix breakage (Fig. 1)) appear nearly uniform throughout the thickness of each mesoconstituent, at least under quasi-static loading. Thus, they can be described in a relatively simple way.

In our method, we conjecture that due to the smallness of the mesoscale (onetenth of a $\mathrm{mm}$ ) a static description of the damage mechanisms should remain valid even for high loading rates. Therefore, we proposing to adapt a mesomodel previously defined for static loading [1-5] to the dynamic case. This mesomodel is initially defined by means of two constituents:

- a single layer which is assumed to be homogeneous and orthotropic,

- $\quad$ an interface, which is a mechanical surface connecting two adjacent layers and which depends on the relative orientation of their fibres (Fig. 1)

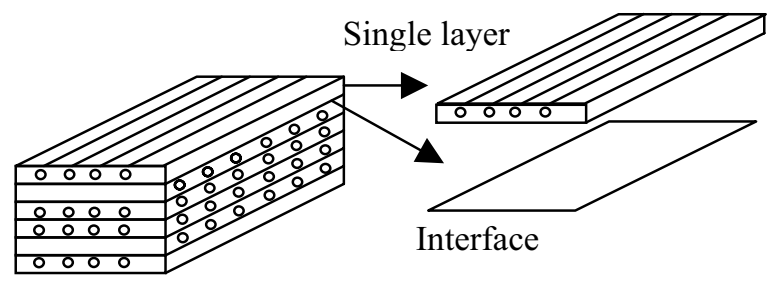

Figure 2: $\quad$ Mesomodel of a laminate.

The damage mechanisms are taken into account by means of internal damage variables. Then, a mesomodel is defined by adding another property, which consists of prescribing a uniform damage state throughout the thickness of the elementary ply.

\subsection{The single-layer model}

The ply is modelled as a homogeneous orthotropic elastic-plastic damaging material whose properties are degraded on loading by microcracking prior to ultimate failure. The first expression used of the strain energy density of damage elementary layer was:

$$
\begin{gathered}
E_{D}=\frac{1}{2}\left[\frac{\left\langle\sigma_{11}\right\rangle_{+}^{2}}{E_{1}^{0}\left(1-d_{1}\right)}+\frac{\left\langle-\sigma_{11}\right\rangle_{+}^{2}}{E_{1}^{0}}-2 \frac{v_{12}^{0}}{E_{1}^{0}} \sigma_{11} \sigma_{22}-2 \frac{v_{13}^{0}}{E_{1}^{0}} \sigma_{11} \sigma_{33}\right. \\
+\frac{\left\langle\sigma_{22}\right\rangle_{+}^{2}}{E_{2}^{0}\left(1-d_{2}\right)}+\frac{\left\langle-\sigma_{22}\right\rangle_{+}^{2}}{E_{2}^{0}}-2 \frac{v_{23}^{0}}{E_{2}^{0}} \sigma_{22} \sigma_{33} \\
\left.+\frac{\sigma_{33}{ }^{2}}{E_{3}^{0}}+\frac{\sigma_{12}{ }^{2}}{G_{12}^{0}\left(1-d_{12}\right)}+\frac{\sigma_{13}{ }^{2}}{G_{13}^{0}}+\frac{\sigma_{23}{ }^{2}}{G_{23}^{0}}\right]
\end{gathered}
$$

where $\langle\rangle+$. designates the positive part. 
This expression enables us to distinguish between tension and compression depending on whether the cracks are closed or open. The rates of release of damage energy associated with $d_{1}, d_{2}$ and $d_{12}$ are expressed as follows:

$$
\left\{\begin{array}{l}
Y_{1}=\left.\frac{\partial\left\langle E_{D}\right\rangle}{\partial d_{1}}\right|_{\sigma}=\frac{\left\langle\left\langle\left\langle\sigma_{11}\right\rangle_{+}^{2}\right\rangle\right\rangle}{2 E_{1}^{0}\left(1-d_{1}\right)^{2}} \\
Y_{2}=\left.\frac{\partial\left\langle E_{D}\right\rangle}{\partial d_{2}}\right|_{\sigma}=\frac{\left\langle\left\langle\left\langle\sigma_{22}\right\rangle_{+}^{2}\right\rangle\right\rangle}{2 E_{2}^{0}\left(1-d_{2}\right)^{2}} \\
Y_{12}=\left.\frac{\partial\left\langle E_{D}\right\rangle}{\partial d_{12}}\right|_{\sigma}=\frac{\left\langle\left\langle\left\langle\sigma_{12}\right\rangle_{+}^{2}\right\rangle\right\rangle}{2 G_{12}^{0}\left(1-d_{12}\right)^{2}}
\end{array}\right.
$$

where $\langle\langle\rangle$.$\rangle designates the mean value through the thickness.$

For the sake of simplicity, the behaviour in the fibre's direction is assumed to be independent on the transverse and shear behaviour. Moreover, through the material parameter $b$, the model introduces a coupling between the evolution of $d_{2}$ and that of $d_{12}$, which, on average, are both associated with the same types of cracks. Then the damage evolution is given by:

$$
\begin{cases}d_{1}=f_{1}\left(\sqrt{\underline{Y_{1}}}\right) & \text { If } d_{1}<1, d_{1}=1 \text { otherwise } \\ d_{2}=f_{2}\left(\sqrt{\underline{Y_{12}+b Y_{2}}}\right) & \text { If } d_{2}<1, d_{2}=1 \text { otherwise } \\ d_{12}=f_{12}\left(\sqrt{\underline{Y_{12}+b Y_{2}}}\right) & \text { If } d_{12}<1, d_{12}=1 \text { otherwise }\end{cases}
$$

where $\underline{Y}=\left.\sup _{\tau<t} Y\right|_{\tau}$ for each quantity $Y$.

The static identification of these evolution laws is carried out by macrotest intension-compression on different stacking sequences of laminate.

Then, in order to model the inelastic strains induced by damage, a plasticity model was built. The elastic domain is defined by the function $f$ such that:

$$
f=\sqrt{\tilde{\sigma}_{11}^{2}+a^{2} \tilde{\sigma}_{22}^{2}}-R(p)-R_{0}
$$

where the threshold $R$ is a function of the accumulated plastic strain $p$; $p \rightarrow R(p)$ is a material characteristic function, and $a^{2}$ is a material characteristic constant. 
With $\stackrel{\sim}{\sigma}$, the effective stress:

$$
\tilde{\sigma}=\left[\begin{array}{l}
\sigma_{11} \\
\frac{\left\langle\sigma_{22}\right\rangle_{+}}{1-d_{2}}+\left\langle\sigma_{22}\right\rangle_{-} \\
\frac{\sqrt{2} \sigma_{12}}{1-d_{12}}
\end{array}\right]
$$

The model assumes that no plastic yield exists in the fibre direction.

\subsection{Interface model}

The interface is simulated as a user contact law between the layer's elements.

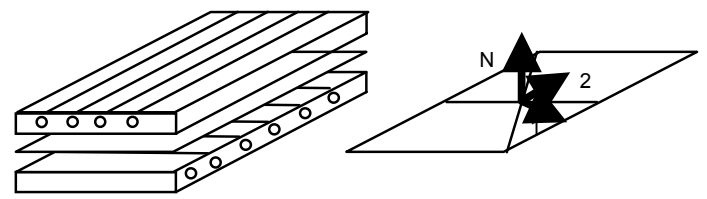

Figure 3: The orthotropic directions of the interface.

This contact law is defined such that:

$$
\begin{aligned}
& \sigma_{n}=K_{n}\left\lfloor\left(1-D_{n}\right)\left(U_{n}^{r}\right)^{+}+\left(U_{n}^{r}\right)^{-}\right\rfloor \\
& \sigma_{c i s 1}=K_{c i s}\left(1-D_{c i s}\right) U_{c i s 1}^{r} \\
& \sigma_{c i s 2}=K_{c i s}\left(1-D_{c i s}\right) U_{c i s 2}^{r}
\end{aligned}
$$

with $U^{r}$ being the relative displacement.

For the interface, the damage evolution is given by

$$
\begin{aligned}
& D_{n}=f\left(U_{n}^{r}\right) \\
& D_{c i s}=f\left(\sqrt{\left(U_{c i s 1}^{r}{ }^{2}+U_{c i s 2}^{r}{ }^{2}\right.}\right) \\
& D_{n}=\max \left(D_{n}, D_{c i s}\right) \\
& D_{c i s}=\max \left(D_{n}, D_{c i s}\right)
\end{aligned}
$$

\section{Simulation of impact}

In order to perform the simulation, the constitutive damage mesomodel was implemented into the explicit finite element code Abaqus/Explicit. To enable the penetration, when $d_{2}$ or $d_{12} \geq 1$, the mesh is eroded. 


\subsection{Delay effect}

A first difficulty with the erosion is the mesh dependency. The following calculation (Figs. 4 and 5.) was performed on a perforating impact of carbon/epoxy layer by a spherical rigid projectile. The problem is solved with the adding of delay effect on damage evolution.

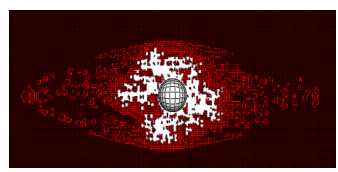

Figure 4: Without delay effect.

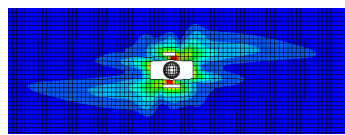

Figure 5: With delay effect.

\subsection{Failure criterion}

With the presented model, calculation didn't be completed because of lot numerical errors, like excessive distortion or time increment fall... To solve these errors, the deformation energy and the erosion criterion were changed. The new deformation energy is:

$$
\begin{aligned}
E_{D}= & \frac{1}{2}\left[\frac{1}{\left(1-d_{1}\right)}\left(\frac{\left\langle\sigma_{11}\right\rangle_{+}^{2}}{E_{1}^{0}\left(1-d_{1}\right)}-2 \frac{v_{12}^{0}}{E_{1}^{0}} \sigma_{11} \sigma_{22}-2 \frac{v_{13}^{0}}{E_{1}^{0}} \sigma_{11} \sigma_{33}\right)+\frac{\left\langle-\sigma_{11}\right\rangle_{+}^{2}}{E_{1}^{0}}+\frac{\left\langle\sigma_{22}\right\rangle_{+}^{2}}{E_{2}^{0}\left(1-d_{2}\right)}+\frac{\left\langle-\sigma_{22}\right\rangle_{+}^{2}}{E_{2}^{0}}\right. \\
& \left.-2 \frac{v_{23}^{0}}{E_{2}^{0}} \sigma_{22} \sigma_{33}+\frac{\sigma_{33}{ }^{2}}{E_{3}^{0}\left(1-d_{2}\right)}+\frac{\sigma_{12}{ }^{2}}{G_{12}^{0}\left(1-d_{12}\right)}+\frac{\sigma_{13}{ }^{2}}{G_{13}^{0}\left(1-d_{1}\right)}+\frac{\sigma_{23}{ }^{2}}{G_{23}^{0}\left(1-d_{2}\right)}\right]
\end{aligned}
$$

With the propagation of the fibre's direction damage to the normal direction, the time increment which is driven by the normal direction increase during the calculation.
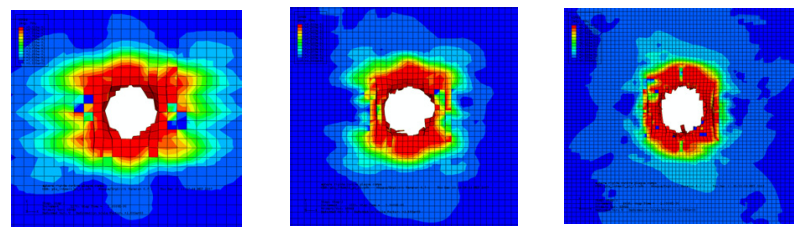

Figure 6: Erosion criterion in the fibre's direction. 
In order to be more consistent and have not numerical difficulties with fibrestocked energy, the failure criterion has been changed. Mesh was eroding only when $d_{1} \geq 1$. A delay effect is introduced for every damage variable.

The new erosion criterion and damage evolution law in the fibre's direction enable to have consistent results in a consistent time with one or more layers.

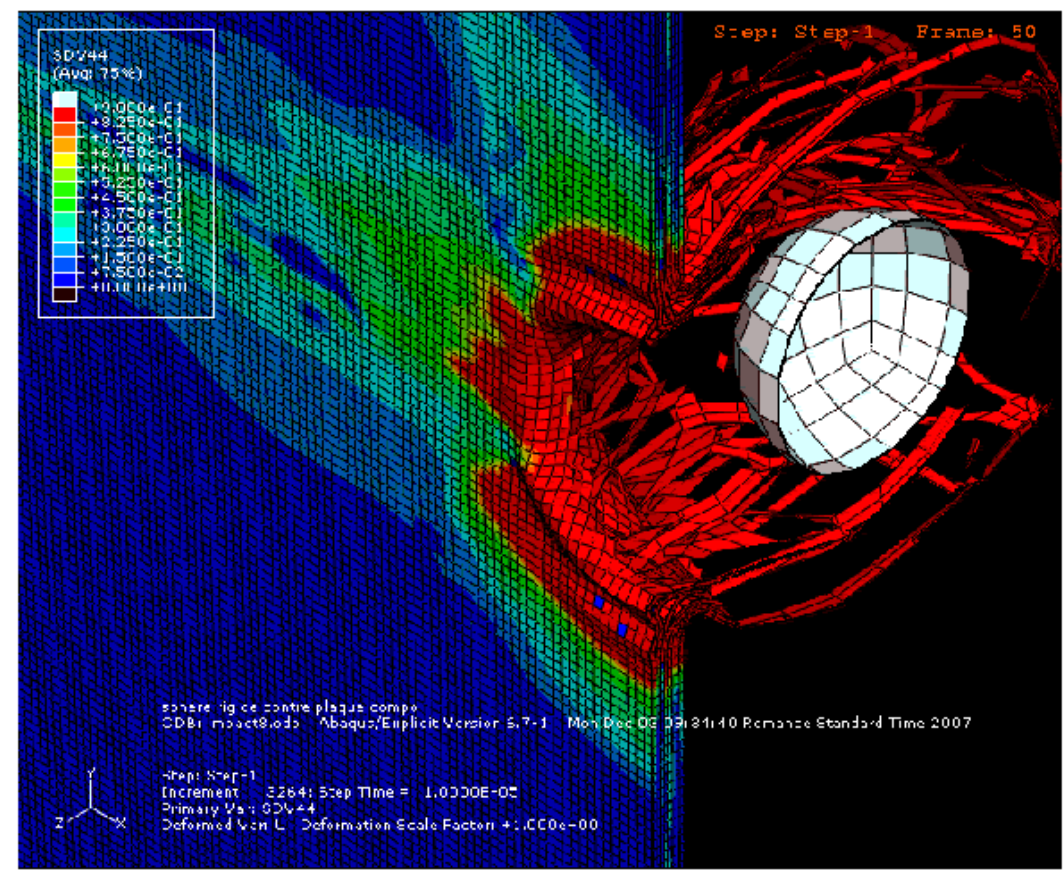

Figure 7: $\quad$ Damage on laminate.

\subsection{First results on laminates}

Calculation mechanical description:

- rigid projectile $10 \mathrm{~g}, 1500 \mathrm{~m} / \mathrm{s}$

- 8 layers, 320000 elements

- 7 contact surfaces of 40000 elements

Calculation numerical description:

- 4 processors

- $2 \mathrm{gb}$ of memory

- $\quad 4 h$ calculation time 
182 Computational Methods and Experiments in Materials Characterisation IV
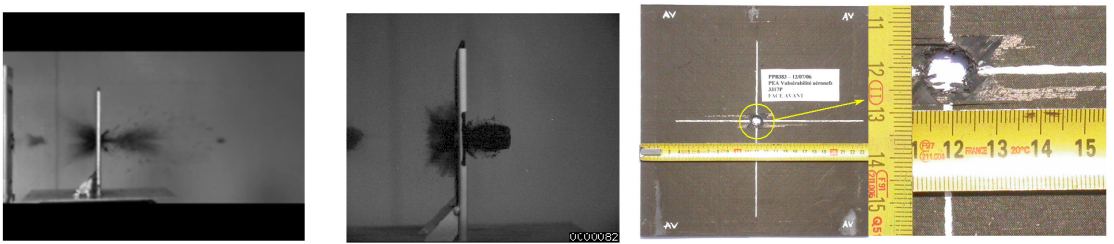

Figure 8: During and after $1500 \mathrm{~m} / \mathrm{s}$ impact.
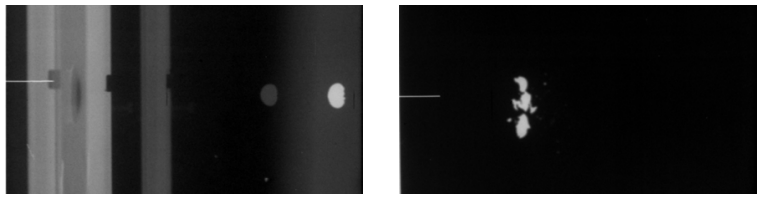

Figure 9: $\quad$ Projectile fragmentation at $3500 \mathrm{~m} / \mathrm{s}$.
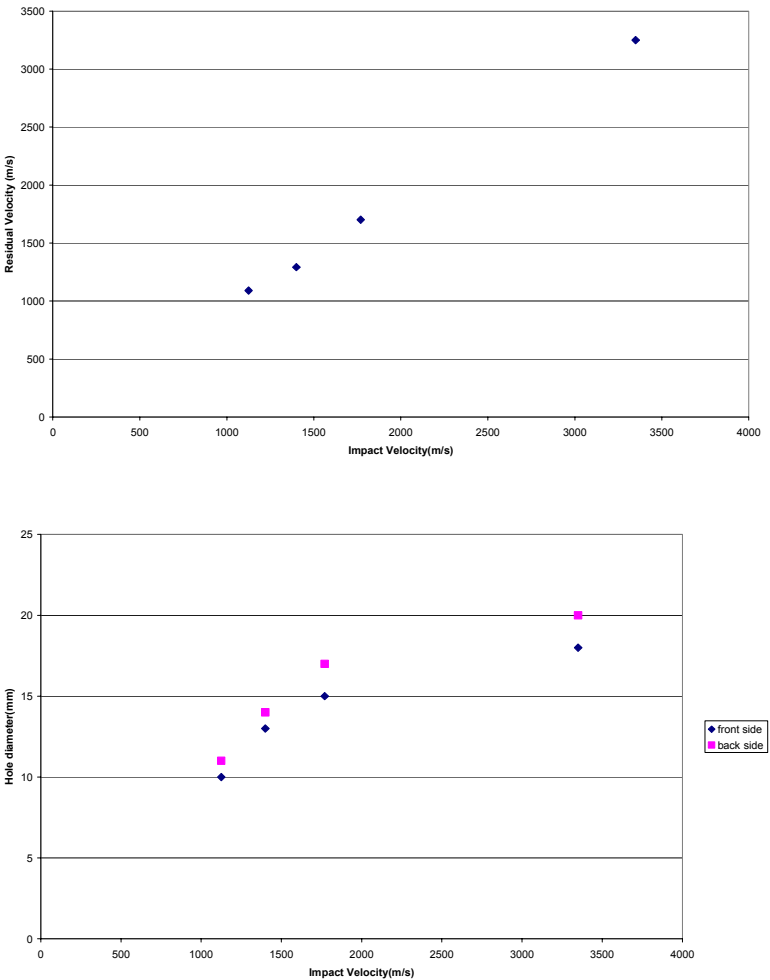

Figure 10: Residual velocity and hole diameter with impact velocity. 


\section{Experimental results}

For velocity ranging from 1000 to $2000 \mathrm{~m} / \mathrm{s}$ the impact tests have been conducted using a powder gun and for velocity ranging 2000 to $3500 \mathrm{~m} / \mathrm{s}$ they have been conducted using the double stage gun Athena in operation at the CEG.

Four tests are presented, for each one the diameter projectile is $10 \mathrm{~mm}$ and the target is thin carbon/epoxy plate.

\subsection{Results}

\subsubsection{Analysis}

The first calculation results are about the same, but CND of plates are waited to have more interested information. We just know at the moment there is nondeceleration of the projectile; the hole's diameter is about the same as the projectile's diameter for $1000 \mathrm{~m} / \mathrm{s}$ and increase with the velocity. An interesting observation is the projectile fragmentation for $3500 \mathrm{~m} / \mathrm{s}$ impact, so we can assume that the physic change between 2000 and $3500 \mathrm{~m} / \mathrm{s}$.

\section{Conclusion and perspective}

In this paper a static mesomodel was adapted to dynamic and penetrating impact. The model presented enable having consistent result in a consistent time. Future works concern a best coupling between different mode in the interface and a best interaction between interface and layer.

Other experimental tests and analysis are pending to validate the numerical results. 